$\begin{array}{ll}\text { Italique } & \text { Italique } \\ \text { Poésie italienne de la Renaissance }\end{array}$

III | 2000

Varia

\title{
Eros e Anteros nella poesia italiana del Rinascimento : appunti per una ricerca
}

\section{Andrea Comboni}

\section{(2) OpenEdition}

12 Journals

Edizione digitale

URL: https://journals.openedition.org/italique/183

DOI: 10.4000/italique.183

ISSN: 1663-4438

\section{Editore}

Librairie Droz

\section{Edizione cartacea}

Data di pubblicazione: 31 décembre 2000

Paginazione: 7-21

ISBN: 2-600-00490-4

ISSN: 1423-3983

Notizia bibliografica digitale

Andrea Comboni, «Eros e Anteros nella poesia italiana del Rinascimento : appunti per una ricerca», Italique [Online], III | 2000, online dal 06 octobre 2009, consultato il 21 septembre 2021. URL: http:// journals.openedition.org/italique/183; DOI: https://doi.org/10.4000/italique.183

(c) Tous droits réservés 
A N D R E A C OM B ON I

EROS E A NTEROS

NELLA POESIA ITALIANA

D E L R I N A S C I M E N T O :

A P P U N T PER UNA RICERCA 


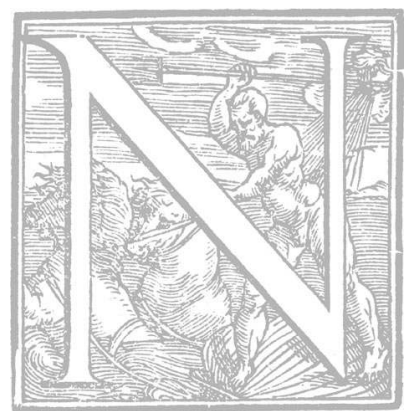

Ell'ultimo decennio ben due convegni hanno richiamato l'attenzione degli studiosi di letteratura rinascimentale sulla figura di Anteros, ${ }^{\mathrm{I}}$ " une figure mythologique quelque peu oubliée et qui réapparait obstinément dans la littérature et l'iconographie à l'aube des temps modernes ${ }^{2}$

Sulla scorta di un noto intervento di Robert $V$. Merrill, ${ }^{3}$ si è cosi cercato di mettere a fuoco le diverse funzioni e $i$ contraddittori significati che in epoca umanisticorinascimentale sono stati attribuiti a questa figura sfuggente e intrigante che, del resto, già nella mitologia e nella tradizione classica risultava priva di una univoca caratterizzazione. ${ }^{4}$ In esse, infatti, poteva rappresentare sia il vendicatore dell'amore disprezzato, sia il patrono dell'amore reciproco, sia il distruttore dell'amore, anche se, nella maggior parte dei casi, "la funzione dell'Anteros classico [...] era stata quella di garantire la reciprocità nelle relazioni amorose »." $N$ ei secoli XV e XVI, come rilevò per primo Erwin Panofsky, si assiste a una moralizzazione di Anteros : " $i$ moralisti e gli umanisti di tendenza platonica erano inclini a interpretare la preposizione antì come 'contro', anziché 'in cambio di', trasformando così il Dio dell' amore reciproco in una personificazione di virtuosa purezza " ${ }^{6}$ la coppia ErosAnteros nella contrapposizione tra due tipi di amore, l'Amore terreno e l'Amore celeste. Non è questa la sede per tracciare la storia del tema dei due amori, l'uno terreno e sensuale, l'altro divino e virtuoso, tema strettamente connesso con la teoria della duplicità di Venere (Ouranìa $e$ Pandemos) che si trova espressa nel Simposio platonico (I80c-I8IC) e da qui prontamente ripresa nel Simposio di Senofonte (VIII 9-10). 7 Come ha ricordato di recente Leandro Ventura, "nella teoria d'amore dell'Umanesimo viene ampiamente recuperata e discussa l'idea platonica della duplicità di Venere e della conseguente duplicità di Amore. Infatti, tale concetto compare già nel I433 in un'epistola inviata da Carlo Marsuppini a Lorenzo Valla [Cod. Riccardiano 779, c. 20Iv]; più tardi, Marsilio Ficino nel suo Commento al Simposio parla a lungo delle due Veneri platoniche; e cosi questa distinzione diventa patrimonio comune del neoplatonismo e si ritrova in numerosi testi 》. ${ }^{8}$ Il tema dei due tipi di amore oltre a conoscere, com'è noto, una significativa fortuna nella pittura quattro-cinquecentesca, è presente pure, in maniera non episodica, nella poesia italiana del Rinascimento. ${ }^{9}$

Tra le rime, recentemente riscattate all'oblio, del mantovano Paride Ceresara, esemplare rappresentante della più alta e raffinata cultura cortigiana tra Quattro e Cinquecento, ${ }^{10}$ si può leggere un sonetto che illustra efficacemente il tema della contrapposizione tra $i$ due tipi di amore: 
Già contemplando in alto i' vedo Amore Nel ciel fra gli altri dèi lucido e chiaro, Dolce, piatoso, stabile e preclaro, Con duo belli occhi e pien d'ogni valore.

Non arco e strali in man, ma di colore

Celeste un lauro tien e quel sì caro Porge ad ognun, perch'è di nulla avaro,

Sol lui mirando e il suo divin splendore. Poi mi rivolgo, e vedo sparsa in terra

Qua l'ombra sua, da lui tutta diversa,

Chiamata Amor, pallida, armata e cieca.

Alora (sento dir) il se disserra

Dal cor un fumo e agli occhi s'atraversa,

Ch'in cotal modo il veder nostro acieca. ${ }^{\mathbf{I}}$

Questo componimento, che avrebbe trovato un'ideale collocazione nel celebre saggio di Panofsky dedicato a Cupido cieco, ${ }^{\mathrm{I} 2}$ descrive nelle quartine l'Amore celeste (splendente, "con duo belli occhi», disarmato), nelle terzine l'Amore terreno (pallido, cieco, armato). ${ }^{13}$ Le quartine mostrano in filigrana qualche ricordo di un famoso epigramma dell'Anthologia Graeca (XVI 20I), attribuito a Mariano Scolastico, nel quale si descrive l'Amore figlio di Venere Urania: ${ }^{14}$

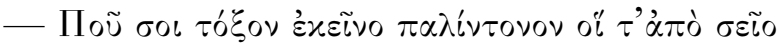

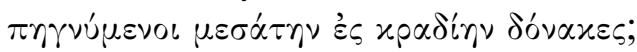

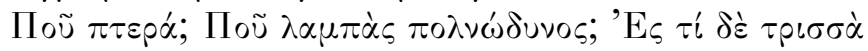

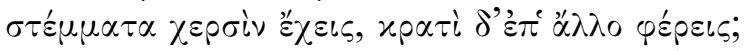

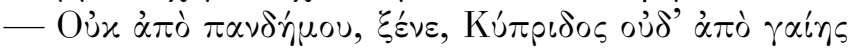

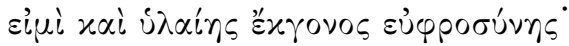

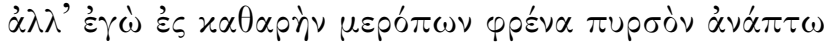

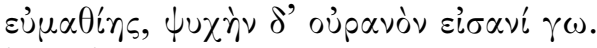

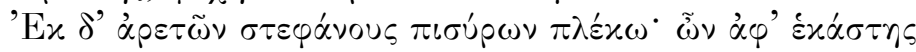

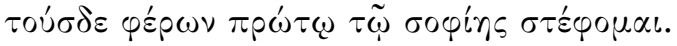

Degno di nota anche il fatto che l'Amore terreno venga presentato quale pallida ombra di quello celeste, cosi come accade nel Commento sopra una Canzona de Amore, composta da Girolamo Benivieni di Giovanni Pico della Mirandola (composto nel I486, diffuso manoscritto, a stampa nel I5I9) : " lo amore vulgare non è vero amore, ma cosi come la sensibile bellezza non è vera bellezza, ma immagine di quella, così è lui uno simolachro e una ombra dello amore celeste $\gg .{ }^{\text {is }}$ 
Non è certo un caso che il Ceresara abbia dedicato un sonetto al tema della contrapposizione tra i due tipi di amore : nella corte di Isabella tale tema era, per cosi dire, all'ordine del giorno, tanto che si ritrova svolto e illustrato nelle tele dei cinque dipinti dello Studiolo. Come ba affermato Giovanni Romano in un saggio per più motivi fondamentale, "il filo che lega l'intera serie [dei dipinti] è un elogio, in cinque capitoli, dell'Amore celeste e delle sue vittorie sull'Amore terreno $\gg{ }^{16}$ E andrà ricordato che Paride Ceresara fu proprio il suggeritore iconografico per alcuni dei dipinti dello Studiolo : sicuramente per la Battaglia di Castità contro Lascivia del Perugino (di cui ci resta la dettagliata invenzione stesa da Paride) e il Regno della Poesia (o della Musica) di Lorenzo Costa, forse anche per il Trionfo della Virtù del Mantegna. ${ }^{17}$

Per quanto riguarda la presenza neoplatonicamente moralizzata di ErosAnteros nella poesia di questa epoca non si può dimenticare come Angelo Poliziano nelle Stanze per la giostra abbia elegantemente svolto questo motivo, facendo della evoluzione di Iulio da una una forma di Eros volgare a quella di un Eros più nobile il "cuore" della storia. Per lo svolgimento di questo tema il Poliziano, secondo la persuasiva dimostrazione di Salvatore Settis, ${ }^{18}$ ha tenuto presente lo stendardo che accompagnava Giuliano de' Medici nel corteo che precedeva la giostra. Questo stendardo mostrava "nella summità un sole», e nel mezzo "una figura grande simigliata a Pallas [...] Teneva decta Pallas nella mano diricta una lancia da giostra et nella mano mancha lo scudo di Medusa》. Accanto a «uno ceppo d'ulivo con uno ramo grande », poi, " era legato uno dio d'amore cum le mani dirieto cum corde d'oro. Et a' piedi aveva archo, turcasso et saecte rocte ». ${ }^{19}$ E sufficiente, credo, questa parziale descrizione per poter affermare come la rappresentazione della Castità che vince Amore presente sullo stendardo di Giuliano e narrata nei versi del Poliziano non sia troppo lontana nello spirito da quella che il Perugino esegui per lo Studiolo : in questa, infatti, avversario di Pallade è, ancora una volta, Cupido. E curiosa la circostanza che, se non ho visto male, non si siano mai citate le Stanze del Poliziano fra i testi letterari che il Ceresara può aver avuto presenti nel comporre l'invenzione per il dipinto del Perugino. Si aggiunga, inoltre, che a Mantova il Poliziano non era certo uno sconosciuto e che Paride fu in relazione con lui. ${ }^{20}$

Esistono anche altri testi poetici rinascimentali in cui viene svolto il tema dei due tipi di amore. La ricerca è appena agli inizi $i$ i componimenti finora rintracciati sono due sonetti e due poemetti in ottava rima.

Il Libro di sonetti et canzoni intitulato Endimione di Benedetto Gareth detto il Cariteo ${ }^{21}$ presenta quasi ad inizio di libro il seguente sonetto: 
Son gemini gli Amori : un casto e pio;

L'altro furente in desiderio insano :

Questo si mostra in terra in volto humano,

Quel vola per li cieli alato idio.

Qual di duo raccendesse il petto mio,

Lo sa colei, che 'l cor mi tiene in mano :

Arsi da presso, e arsi di lontano,

Con la speranza eguale al gran desio.

Con violenta voglia e importuna,

Anhelando al sidereo, almo paese,

Servii senza cercar mercede alcuna.

Celeste fu la fiamma che m'accese :

Ché di quelle, che in ciel movon la luna,

Una angelica forma il cor mi prese. ${ }^{22}$

La contrapposizione tra $i$ due tipi di amore, compendiosamente esposta nella prima quartina, diventa a partire dal $v$. , l'occasione per tessere un galante elogio della donna amata (petrarchescamente definita " una angelica forma »). Anche tra le rime di Francesco Beccuti detto il Coppetta ${ }^{23}$ s'incontra un sonetto che, in modo più articolato e meno occasionale rispetto al Cariteo, tratta il nostro tema:

Voi ch'ascoltate l'una e l'altra lira

Degli onorati duo tra noi migliori,

Sapete ben che con diversi ardori

Lalage questi e quei Laura sospira,

E che colei che 'l terzo cielo gira,

Fu qua giù madre di gemelli Amori,

E ch'ambo pronti ad impiagare i cori,

L'uno vil voglie e l'altro oneste inspira.

A che col volgo dite :- Un arcier solo

Punge ogni petto, e va sotto un'insegna

Socrate ancor fra l'amoroso stuolo? -

Crediate omai che chi nel mio cuor regna

Non è nudo né cieco, e col suo volo

Di levarmi da terra ognor m’insegna. ${ }^{24}$

Il sonetto in questione sembra costituire il frammento di un discorso sulla natura di amore nel quale il Coppetta si inserisce rivendicando con decisione e fermezza la duplicità di Cupido ("gemelli Amori", cosi come nel Cariteo "gemini [...] Amori», figli dunque di un'unica Venere). Da segnalare che il v. 6 è traduzione quasi letterale dell'incipit del IV libro dei Fasti ovidiani ("Alma fave dixi geminorum mater amorum ») e che al $v$. I3 si sottolinea la 
non nudità e la non cecità dell'amore onesto e virtuoso. L'ultima terzina consuona singolarmente con la corrispondente del sonetto del Cariteo.

Il tema dei due amori registra infine un'udienza particolare presso Antonio Fileremo Fregoso, poeta legato, dapprima, alla corte sforzesca, della quale, come testimonia il Calmeta, costituiva insieme a Niccolò da Correggio e Gasparo Visconti, il più insigne ornamento, poi, dopo la caduta di Ludovico il Moro, fedele ai francesi. ${ }^{25}$ Nella Cerva bianca, poemetto allegorico in ottave diviso in sette canti, pubblicato per la prima volta a Milano nel I $510,{ }^{26}$ il Fregoso racconta la vicenda della ninfa Mirina tramutata da Diana in fiera e del cacciatore Fileremo (cioè il poeta stesso) lanciato al suo inseguimento con $i$ cani Desio e Pensiero, nel tentativo di restituirle figura umana, attraverso $i$ regni di Diana, d'Antero e d'Amore. L'intera narrazione si caratterizza per "il gusto della gradualità che conduce dalla passione all'esperienza intellettuale dell' amore divino, tipico della linea speculativa neoplatonica. E l'affermazione non si riferisce tanto alla patina che caratterizza la trattatistica antierotica settentrionale fra Quattro e Cinquecento (valgano $i$ nomi del Platina, di Battista Fregoso, di Petrus Haedus e del Corio), quanto piuttosto alla fortuna, di qua dall'Appennino, di testi celebri come il Comentarium in Convivium Platonis di Marsilio e il Commento di Pico alla Canzona de Amore di Girolamo Benivieni $\gg{ }^{27} \dot{E}$ innanzitutto il nome di Antero a richiamare la nostra attenzione: assente nei testi poetici finora ricordati, viene impiegato dal Fregoso per indicare, sulla scorta di un passo del cicerioniano De natura deorum (III 23, 59-60), il figlio adulterino di Venere e Marte :

Io credo certo che ambidoi sappiate come Venere e Marte da Vulcano fur presi ne le reti fabricate a questo effetto de sua propria mano, e se dil tutto ben vi reccordate, scoperti furno a quel stroppiato e strano dal radiante gran signor di Delo, che fabula non è più nota in cielo.

De lo adultero amante e vulgar dea un figlio nacque nominato Antero ; e perché patre bellicoso avea, tutto marziale e furibondo e fiero è nato ; e perché Amor signor vedea in sì gran stato naturale e vero, per esser figlio del spietato Marte, per forza gli n'ha tolto una gran parte. 


\section{Andrea Comboni}

Pel regno iniusto di quel gran tiranno noi passarem non senza gran periglio : i soi sugetti sempre in guerra stanno, ché dove è tal furor, non è consiglio. Ma se passar desiate senza danno, né far volete quel terren vermiglio dil vostro sangue, pronti a me attendete, e tutto quel ch'io dico osservarete.

(Cerva bianca, $\mathrm{V}$ 37-39) ${ }^{28}$

Ciò che più sorprende in queste ottave (in cui a parlare è la Ragione) e che invano si cercherebbe nel brano ciceroniano, è la connotazione fortemente negativa attribuita ad Antero, "tutto marziale e furibondo e fiero », " gran tiranno ", irriducibile avversario di Amore, a cui con la forza ha sottratto "una gran parte » del suo originario dominio. Nel regno del "crudo Antero », dove i reggitori sono Furore, Sdegno e Gelosia, ${ }^{29}$ gli abitanti « son furibondi e insani » (V 6I, 5). Il dominio di Antero è descritto quindi come il dominio delle passioni irrazionali. I regni di Antero e di Amore sono divisi da un fiume che trae la sua origine dalle lacrime degli infelici sudditi del «crudo Antero »:

Questo è quel fiume che divide il regno

dil crudo Antero dal più culto stato

de Amor, quale è signor potente e degno.

(Cerva bianca, VI I 2, I-3)

Nel regno di Amore ha sede Erotopoli, alla sommità della quale si trova il " templo » del « divo Amore [...] creato in la celeste sede » (VII 65, 6 e 8), all'interno del quale il cacciatore (Fileremo, cioè il Fregoso) è introdotto dalla Ragione :

Approssimati al principale altare, in una chiara luce io vidi Amore, né offesa fu mia vista nel mirare, ché era purgata al fonte vivo fuore. Ragion mi fece allora ingenochiare supplice inante a quel divino ardore, e vòlta a me diceva : «Ho satisfatto al mio parere ad ogni nostro patto.

Ecco qua Amore in la sua eterna sede, ecco te ho appresentato al suo cospetto, 
qual di chiarezza ogni altra luce eccede. Mandato ho le promisse mie ad effetto, de le qual mi obbligai allor per Fede.

Questo è quel vero Amor sincero e netto, al qual menarti sempre mai intesi, quando scioglier ve feci, essemdo presi.

Mira che da' bei fianchi non gli pende faretra alcuna, e lo arco e stral pongente in man non ha, perché niuno offende. In la man destra ha sol la face ardente, con quale a la virtù la anima accende, poi l'alza al ciel, tanto è suo ardor potente. Quattro corone in la sinistra tiene, che son premio a ciascun chi 'l serve bene.»

(Cerva bianca, VII 72-74)

La descrizione del « vero Amor sincero e netto » privo di faretra, arco e strali, con una fiaccola nella mano destra e quattro corone nella sinistra serba evidente memoria dell'epigramma dell'Anthologia Graeca attribuito a Mariano Scolastico e qui già ricordato quale probabile fonte del sonetto di Paride Ceresara sui due tipi di Amore. Degno di nota resta il fatto che nella Cerva bianca si assiste ad una sorta di rovesciamento delle funzioni tra Eros e Anteros, che fa del secondo il polo negativo della contrapposizione.

A una quindicina d'anni dall'editio princeps della Cerva bianca, nella III delle sue Silve, ${ }^{30}$ intitolata Pergoletta de le laudi de Amore $e$ composta nel metro dell'ottava, il Fregoso, dialogando con gli amici Bartolomeo Simonetta e Antonio Telesio, discute e contesta la tradizionale rappresentazione di Amore come "cieco, nudo fanciul, con pennate ali [...] con l'arco in mano e con faretra e strali " (III 3, I-3), concludendo che questa iconografia non si addice all" "Amor sacro e celeste » (III 3, 2), ma all" "Amor vulgare e osceno »!

Qual altra cosa in questa mortal vita è più salubre a un giovenetto core ch'arder per donna de virtù munita? Ogni tristo pensier quel sacro ardore scaccia dal petto giovenil e invita l'animo sempre a sequitar onore : se lo amante in l'amata se trasforma, i bei costumi suoi tien per sua norma. 
Ma chi femina vil per sorte mira, ch'abbia l'anima infetta, quel veneno a sé, com'occhio d'occhio infermo, tira e de vani pensieri gli empie il seno, per quale avvien che poi piange e suspira : chiamasi questo Amor vulgare e osceno, a cui ben gli conviene l'arco e i strali, perch'è sola cagion de tutti i mali.

Del nostro sacro e venerando nume che direm noi, da poi che solo è quello ch'ha in sé ogni grazia e ogni bon costume? A chi 'l depinse giovenetto e bello benedico la man, ma chi prosume cieco chiamarlo e de pietà rebello, esso è profano e cieco e senza legge, ch'ha le sue luci e in pace il mondo regge.

(Pergoletta de le laudi de Amore, i 8-20)

L'intero poemetto vuole essere, come promette il titolo, una lode d'Amore, più precisamente, una lode dell" "Amor sacro e celeste », dio di pace e di concordia, fonte di virtù e di elevazione morale. In termini platonici (e neoplatonici) se ne descrive la genesi, per poi introdurre sùbito la distinzione, più che la contrapposizione, di due forme di Eros, una celeste, l'altra invece terrena:

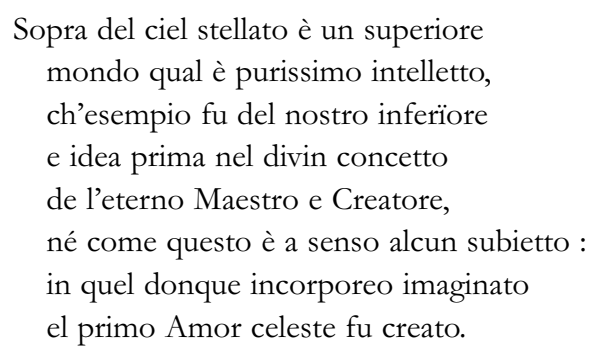

Però convien ch'in questo inferior mondo gli regni un altro Amor simil a quello, il qual chiamar debbiamo Amor secondo, più ch'alcun'altra creatura bello ; sì come quello Amor fa 'l ciel giocondo, così fa in terra questo suo fratello : quello de l'alma la bellezza affetta questo in quella del corpo se deletta. 
Secondo il Fregoso, tra queste due forme di Amore, che paiono ricalcare in parte le caratteristiche dell'uno e dell'altro Eros del Simposio platonico, ${ }^{31}$ non deve esservi contrapposizione ma collaborazione, non contesa ma concordia:

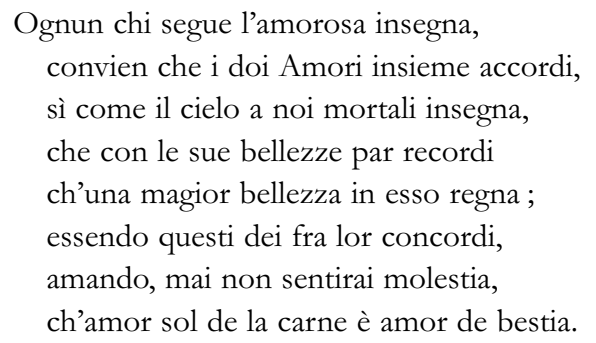

(Pergoletta de le laudi de Amore, 44)

I componimenti poetici fin qui ricordati attestano, come s'è visto, svolgimenti non sempre univoci del tema dei « doi Amori $»$. Del resto se si volge lo sguardo alle testimonianze dell'arte rinascimentale si registrano risultati analoghi. ${ }^{32}$ Anche da una prima verifica compiuta sui coevi commenti ai poeti classici o a Petrarca, che contengono talvolta testimonianze generalmente trascurate della fortuna quattro-cinquecentesca di Eros-Anteros, risulta come la duplicità di Amore venga interpretata e spiegata secondo diverse sfaccettature. Il bolognese Filippo Beroaldo nel suo commento a Properzio I I I cosi scrive :

Plato in Symposio scribit duos esse cupidines alterum celestem et diuinum, alterum uulgarem atque plebeium. Quod secutus uidetur Ouidius in Fastis sic scribens: Alma faue dixi geminorum mater amorum. Greci Erota amorem appellant [...]. Non pretereundum est deum, qui amori contrarius est amoremque dissoluit uocitari Anterota: de quo intellegit Cicero sic scribens in tertio De natura deorum : ex Venere et Marte natus Anteros dicitur : eiusdem meminit et Seruius in Quarto commentario. ${ }^{33}$

\section{Antonio Costanzi da Fano chiosa in questo modo Ovidio, Fasti $I V I$ :}

Dicitur autem Venus geminorum cupidinum mater, sed scripserunt quidam plures esse Veneres pluresque cupidines: ut Cice. qui duas Veneres tradit \& tres Cupidines. Primum ex Mercurio \& Diana genitum. Secundum ex Mercurio \& secunda Venere. Tertium quem Anteron dicunt. [...] Plato duplicem amorem esse dixit : alterum terrestrem atque vulgarem, alterum caelestem singularem ac plane divinum, \& hunc quidem esse omni studio ac diligentia expetendum, illum uitandum tanquam humano generi perniciosum. idemque hunc caelestem amorem fauorem etiam uocat, sed sanctum ac diuino quodam instinctu prouenientem. ${ }^{34}$ 
I due tipi di Amore possono venire ricordati anche nell' inizio di un commento al petrarchesco Triumphus Cupidinis $I$ :

Si fingono esser' due Venere, vna celeste \& vna terrestre, e dui amori, vno diuino e l'altro humano. Del divino ne ragionò copiosissimamente il Pico nella canz. del Beniuieni : dell'altro Guido Cavalcanti nella sua famosa canz. Gl'effetti del primo sono in ogni parte lodeuoli, del secondo biasimevoli; per il primo l'huomo si lieua, da queste cose basse, alla contemplatione delle cose superne, per il secondo l'huomo getta Idio dopo le spalle e s'immerge in questi affetti terreni applicandosi alle cose disordinate. Il primo fa star sopite tutte le potentie appetitiue, il secondo le cognitiue. Di questo secondo volgare $\&$ inhonesto tratta il nostro poe. e mostra con essempi, infiniti essere quelli che hanno seguito il senso, conculcata la ragione. ${ }^{35}$

Andrea Comboni 


\section{Eros e Anteros}

I. Ci si riferisce a Eros and Anteros: The Medical Traditions of Love in the Renaissance, Edited by D. A. Beecher and M. Ciavolella, Toronto, 1992 e ad Anteros, Actes du colloque de Madison (Wisconsin), sous la direction de U. Langer et J. Miernowski, Orléans, I994.

2. J. Miernowski, Anteros, face à face, in Anteros cit., p. I 2.

3. R. V. Merrill, Eros and Anteros, «Speculum », I9 (1944), pp. 265-84; questo saggio è stato ripubblicato, tradotto in francese, in Anteros cit., pp. 27-59 (da cui si cita).

4. Cfr. R. V. Merrill, Eros et Anteros, in Anteros cit., pp. 29-39.

5. E. Panofsky, Studi di iconologia. I temi umanistici nell'arte del Rinascimento, Introduzione di

G. Previtali, Traduzione di R. Pedio, Torino, I975, p. I77.

6. E. Panofsky, Studi di iconologia cit., pp. 177-78.

7. Sulla teoria platonica della duplicità di Venere (e di Amore) si vedano S. Settis, Cheloné. Saggio sull'Afrodite Urania di Fidia, Pisa, 1966, pp. 97-107 ; J. Rudhardt, Eros e Afrodite, Torino, I999, pp. $25-6$.

8. L. Ventura, Lorenzo Leonbruno. Un pittore a corte nella Mantova di primo Cinquecento, Roma, I 995, p. Iо . Fondamentali osservazioni sulle «Due Veneri » (o «Veneri gemelle ») e sui due Amori in ambito neoplatonico si leggono in E. Panofsky, Studi di iconologia cit., pp. 196-235.

9. Per quanto riguarda la letteratura italiana quattro e cinquecentesca, le indagini sui diversi tipi di amore privilegiano, com'è naturale, la produzione trattatistica di argomento erotico, cfr., ad esempio, M. Ciavolella, Trois traités $d u X V^{e}$ siècle italien sur Anteros : "Contra amores » de Bartolomeo Sacchi, "Anterotica» de Pietro Edo et «Anteros» de Battista Fregoso, in Anteros cit., pp. 61-73; A. Quondam, Sull'orlo della bella fontana. Tipologie del discorso erotico nel primo Cinquecento, in Tiziano. Amor Sacro e Amor Profano, Milano, i 995, pp. 65-8 I.

Io. Su Paride Ceresara, insigne figura di umanista che animò la vita artistica e culturale della corte mantovana di Isabella d'Este, cfr. A. Comboni, Paride Ceresara, mantovano, in Veronica Gambara e la poesia del suo tempo nell'Italia settentrionale. Atti del Convegno (Brescia-Correggio, 17-19 ottobre 1985), a c. di C. Bozzetti, P. Gibellini, E. Sandal, Firenze, 1989, pp. 263-80; P. Porçal, Due lettere sulle imprese di casa Gonzaga. Contributo alla prassi pre-accademica delle prime imprese italiane, "Mitteilungen des Kunsthistorischen Institutes in Florenz», 40 (1996), pp. 233-35.

I I. Si cita da A. Comboni, Le rime di Paride Ceresara : edizione e commento, Tesi di dottorato di ricerca in Filologia romanza e italiana, III ciclo, Università degli Studi di Roma «La Sapienza », pp. 185-87.

I 2. E. Panofsky, Studi di iconologia cit., pp. I 35-83.

I 3. Come ha osservato Robert D. Cottrell, Le déplacement d"Eros par Anteros dans "L'Amye de Court» de La Borderie, in Anteros cit., pp. I 26-27 : «La différence entre Eros et Anteros peut être suggérée emblematiquement par le fait qu'Eros est aveugle, alors qu'Anteros ne l'est pas ».

14. Antologia Palatina, a c. di F. M. Pontani, Volume IV, Torino, I98 I, pp. 356-59 (traduzione: "-Dove l'elastico arco finì ? Dove sono le frecce / che la tua mano in mezzo al cuore infisse? / Dove le ali ? e la face così dolorosa ? E a che scopo / le tre corone in mano 


\section{Andrea Comboni}

e in capo l'altra? / - Ospite, non da Ciprigna pandemia discendo, e non nacqui / dalla terra o da gioie materiali. / Ardo negli animi puri del retto sapere la face / e conduco lo spirito nel cielo. / Sono di quattro virtù le corone che intreccio, e sapienza / mi dà, dei serti onde mi cingo, il primo »). La traduzione in latino di questo epigramma accompagna l'emblema CX (Anteros, id est Amor virtutis) di Andrea Alciato (cfr. Emblemata. Handbuch zur Sinnbildkunst des XVI. und XVII. Jabrbunderts, herausgegeben von A. Henkel und A. Schone, Stuttgart, 1967, coll. 1767-68).

i s. Giovanni Pico della Mirandola, Commento sopra una canzone d'amore, a cura di P. De Angelis, Palermo, 1994, p. 9r. Si ricordi come negli Asolani (II xv) Perottino venga definito da Gismondo «ombra [...] d'amante, più tosto che amante » (Prose e rime di Pietro Bembo, a cura di C. Dionisotti, Torino, $1966^{2}$, p. 410.

I6. G. Romano, Verso la maniera moderna : da Mantegna a Raffaello, in Storia dell'arte italiana, 6/1, Torino, I981, p. 38.

17. Cfr. E. Verheyen, The Paintings in the Studiolo of Isabella d'Este at Mantua, New York, I 97 I, pp. 22-9, 4I-6; Le Studiolo d'Isabelle d'Este, Catalogue rédigé sous la direction de S. Béguin par C. Adelson, J. Biscontin, M. A. Debout, L. Fallay-d'Este, M. H. Girard, A. Mérot, J. Schloder, Paris, I 975, pp. 4I-4, 49-5 I ; G. Romano, Verso la maniera moderna cit., pp. 30-I ; K. Christiansen, Lo Studiolo di Isabella d'Este e i temi tardi, in Andrea Mantegna, a c. di J. Martineau, Milano, 1992, pp. 422-23, 426; «La prima donna del mondo». Isabella d'Este. Furstin und Mazenatin der Renaissance, Wien, 1994, pp. 222-38 ; A. Cole, La Renaissance dans les cours italiennes, Paris, I995, pp. 165, I67, 169.

I8. S. Settis, Citarea 'su una impresa di bronconi', «Journal of the Warburg and Courtald Institute», 34 (I97I), pp. I 35-77.

I9. S. Settis, Citarea cit., p. I40.

20. Cfr. A. Comboni, Paride Ceresara cit., p. 268 n. I9.

21. Sul Cariteo si veda l'eccellente monografia di G. Parenti, Benet Garret detto il Cariteo. Profilo di un poeta, Firenze, 1993 ; cfr. anche M. Santagata, La lirica aragonese. Studi sulla poesia napoletana del secondo Quattrocento, Padova, 1979, pp. 296-34I.

22. Le rime di Benedetto Gareth detto il Chariteo secondo le due stampe originali, con Introduzione e note di E. Pèrcopo, II, Napoli, i 892, p. 8. Il Pèrcopo pubblica l'Endimione secondo la stampa del i 509 (Napoli, S. Mayr).

23. Sul Coppetta cfr. la voce di C. Mutini nel Dižionario biografico degli italiani, 7 , Roma, 1965 , pp. $498-502$.

24. Giovanni Guidiccioni-Francesco Coppetta Beccuti, Rime, a cura di E. Chiorboli, Bari, I91 2, p. I 28

25. Sul Fregoso cfr. G. Dilemmi, Di un poeta «milanese» fra Quattro e Cinquecento : Antonio Fileremo Fregoso, in Studi di filologia e di letteratura italiana offerti a Carlo Dionisotti, Milano-Napoli, 1973, pp. I17-35. La testimonianza del Calmeta si legge ne La vita di Serafino Aquilano: «Ornavano quella corte tre generosi cavallieri, li quali, oltra la poetica facultate, di molte altre virtù erano insigniti : Nicolò da Correggio, Gasparro Vesconte, Antognetto da Campo Fregoso » (Vincenzo Calmeta, Prose e lettere edite e inedite (con due appendici di altri inediti), a c. di C. Grayson, Bologna, i959, p. 71). 


\section{Eros e Anteros}

26. Antonio Phileremo Fregoso, Cerva bianca, Impresso in la Inclita città de Milano, per Petro Martire di Mantegazzi dicto il Cassano ad instantia de Dominico da la Piaza del Degno Authore amanuense, is ro. Quest'opera, ritenuta il capolavoro del Fregoso, avrà nell'arco di circa mezzo secolo altre undici edizioni : l'ultima nel i 566 (Venezia, F. Rampazetto).

27. G. Dilemmi, Di un poeta «milanese » cit., pp. I 32-33.

28. Questo e i seguenti brani si citano da Antonio Fileremo Fregoso, Opere, a c. di G. Dilemmi, Bologna, 1976 .

29. La Gelosia, si leggerà più avanti, «non appartiene ad Amor vero, / germana è certo dil fallace Antero » (VI 36, 7-8).

30. L'editio princeps delle Silve del Fregoso fu pubblicata con il titolo di Opera nova, In Milano, per Bartolomeo da Crema Ad instantia de Messer Ioanne Iacobo \& fratelli de Legnano, I 525. Questa edizione ebbe in seguito un'unica ristampa nel i 528 (Venezia, N. Zopino).

3I. I vv. 7-8 dell'ott. 34 sintetizzano definizioni presenti nel Commento sopra una canzone d'amore del Pico : «l'amore volgare, cioè della bellezza corporale [...] L'opposito è nello amore celeste, nel quale [...] tutto tende e si diriza alla bellezza spirituale dello animo e dello intelletto » (ed. cit., pp. $9^{\mathrm{I}-2)}$.

32. Cfr., ad esempio, E. Verheyen, Eros and Anteros : «L'education de Cupidon» et la prétendue "Antiope » du Corrège, «Gazette des Beaux-Arts ", 65 (1965), pp. 32 I-40; G. de Tervarent, Eros and Anteros, or reciprocal Love in ancient and Renaissance Art, «Journal of the Warburg and Courtald Institutes », 28 (1 965), pp. 205-8; L. Ventura, Lorenzo Leonbruno cit., pp. 98-105, i Io- I 5, i 87-88; Tiziano. Amor Sacro e Amor Profano cit., passim. Si ricordino anche i celebri emblemi CX (Anteros, id est Amor virtutis) e CXI (Anteros, Amor virtutis, alium Cupidinem superans) dell'Alciato, cfr. Andreae Alciati, Emblemata cum commentariis Claudii Minois [...], Patavij, apud Petrum Paulum Tozzium, I621, pp. 457-65.

33. Sextus Aurelius Propertius, Elegiae, comm. Philippus Beroaldus, Bologna, Platone de' Benedetti, ed. Benedetto Faelli, I487, c. a IIIIr. Sul Beroaldo si veda la voce di M. Gilmore nel Dizionario biografico degli italiani, 9, Roma, 1967, pp. 382-84. Nella trascrizione di questo, come dei brani successivi, mi sono limitato ad ammodernare l'interpunzione e l'uso delle maiuscole.

34. Publius Ovidius Naso, Fasti, comm. Antonius Constantius, Venezia, Giovanni Tacuino, pr. id. iun. [I 2 VI] I 497, c. cxxvirr. Sul Costanzi cfr. la voce di G. Formichetti nel Dižionario biografico degli italiani, 30, Roma, I984, pp. 370-74.

35. Il Petrarcha col commento di M. Sebastiano Fausto da Longiano, con rimario et epiteti in ordine d'alphabeto nuovamente stampato, Stampato in Vinegia a san Moyse, al segno dell'Angelo Raphael, per Francesco di Alessandro Bindoni, e Mapheo Pasini, compagni. Ne gl'anni del nostro Signore M.D.xxx.II, c. 242v. Su questo commentatore petrarchesco cfr. G. Belloni, Laura tra Petrarca e Bembo. Studi sul commento umanistico-rinascimentale al «Canzoniere», Padova, I992, pp. I 20-45. 